\title{
ON DERIVATION ALGEBRAS OF MALCEV ALGEBRAS AND LIE TRIPLE SYSTEMS
}

\author{
ERNEST L. STITZINGER
}

\begin{abstract}
W. H. Davenport has shown that the derivation algebra $\mathscr{D}(A)$ of a semisimple Malcev algebra $A$ of characteristic 0 acts completely reducibly on $A$. The purpose of the present note is to characterize those Malcev algebras which have such derivation algebras as those whose radical is central and to obtain the same result for Lie triple systems. Analogous results are known to hold for standard and alternative algebras.
\end{abstract}

TheOREM. Let $A$ be a Malcev algebra or a Lie triple system over a field of characteristic 0 . Then the derivation algebra $\mathfrak{D}(A)$ of $A$ acts completely reducibly on $A$ if and only if the radical $R$ of $A$ is contained in the center $Z$ of $A$.

All algebras considered here are finite dimensional over a field of characteristic 0 and the unexplained notation is as in [8]. We note that if $S$ is a subset of a vector space $A$, then $\langle S\rangle$ denotes the linear span of $S$.

REMARK. The referee has noted that for Malcev algebras the condition that the radical is central is characterized in several ways in [5, Lemma 3].

1. The Malcev algebra case. An algebra $A$ is said to be Malcev if the identities

$$
x^{2}=0
$$

and

$$
(x y)(x z)=((x y) z) x+((y z) x) x+((z x) x) y
$$

are satisfied in $A$. Sagle [7, p. 453] has shown that for each $x, y \in A, D(x, y)$ $=\left[R_{x}, R_{y}\right]+R_{x y}$ is a derivation of $A$ and that if $D \in \mathfrak{D}(A)$, then

$$
[D(x, y), D]=D(x D, y)+D(x, y D) \text {. }
$$

A simple computation shows that

$$
J(x, y, z)=x D(y, z)+y D(z, x)+z D(x, y)
$$

holds in $A$ where $J(x, y, z)=(x y) z+(y z) x+(z x) y$.

We recall some definitions for a nonassociative algebra $A$. Let $A^{(1)}$ $=A$ and $A^{(n+1)}=\left(A^{(n)}\right)^{2}$ for $n=1,2, \ldots$ Then $A$ is said to be solvable if $A^{(r)}=0$ for some integer $r$. In general, $A^{(i)}$ may not be an ideal of $A$. For many classes of algebras, including Malcev algebras, one obtains a series of

\footnotetext{
Received by the editors October 22, 1974 and, in revised form, January 16, 1975.
} AMS (MOS) subject classifications (1970). Primary 17A30. 
ideals of $A$ as follows: Let $B$ be an ideal of $A$, let $B^{[1]}=B$ and let $B^{[n+1]}=B^{[n]} B^{[n]}+\left(B^{[n]} B^{[n]}\right) A$. If $A$ is Malcev, then $B^{[n]}$ is an ideal of $A$ for each integer $n$. If $B^{[r]}=0$ for some $r$, then $B$ is called an $L$-solvable ideal of $A$. By [6, Theorem 1.1] solvability and $L$-solvability are equivalent notions for ideals of Malcev algebras. Now suppose that $B$ is a minimal solvable ideal of $A$. Then $B^{[2]}$ is an ideal of $A$ properly contained in $B$; hence, $B^{[2]}=0$ and, hence, $B^{2}=0$. A Malcev algebra is called abelian if $A^{2}=0$, and since $A$ is anticommutative, $A$ is abelian if and only if it is commutative. Likewise an ideal of a Malcev algebra is central if and only if it is an annihilating ideal. An algebra $A$ is called nilpotent if there exists an integer $t$ such that every product of $t$ elements of $A$, no matter how associated, is 0 . Clearly if $A$ is nilpotent, then $A$ is solvable, but the converse fails for Malcev algebras. Finally, the sum of two nilpotent ideals of a Malcev algebra $A$ is a nilpotent ideal; hence, there exists a maximal nilpotent ideal called the nil-radical of $A$.

Proof of Malcev algebra Case. Suppose that $A$ is a Malcev algebra and that $R \subseteq Z$. Under this assumption, $R$ is complemented by a semisimple subalgebra $S[10$, Theorem 1]. Let

$$
\begin{aligned}
& \mathfrak{D}_{1}=\{D \in \mathfrak{D}(A) ; D: S \rightarrow S, D: R \rightarrow 0\} \text { and } \\
& \mathfrak{D}_{2}=\{D \in \mathfrak{D}(A) ; D: S \rightarrow 0\} .
\end{aligned}
$$

We claim that $\mathfrak{D}(A)=\mathfrak{D}_{1} \oplus \mathfrak{D}_{2}$ and that $\mathfrak{D}_{1}$ and $\mathfrak{D}_{2}$ are completely reducible acting on $A$. Since $\mathfrak{D}_{1}$ and $\mathfrak{D}_{2}$ are then ideals of $\mathfrak{D}(A)$, the result will then follow. Let $D \in \mathfrak{D}(A)$ and $x \in S$. Then $x D=(x D)_{S}+(x D)_{R}$ where $(x D)_{S}$ $\in S$ and $(x D)_{R} \in R$. If $y \in S$, then

$$
((x y) D)_{S}+((x y) D)_{R}=(x D)_{S} y+(x D)_{R} y+x(y D)_{S}+x(y D)_{R} .
$$

Define $D_{S}$ and $D_{R}$ on $S$ by $(x) D_{S}=(x D)_{S}$ and $(x) D_{R}=(x D)_{R}$. Then $D_{S}$ is a derivation of $S$ into $A, D_{R}$ is a derivation of $S$ into $R$ and $\left.D\right|_{S}=D_{S}+D_{R}$. By [10, Lemma 2], $D_{R}$ can be extended to a derivation of $A$ of the form $\sum D\left(x_{i}, y_{i}\right)$ where $x_{i} \in R, y_{i} \in S$. Then, since $R \subseteq Z, D_{R}=0$. Then $\left.D\right|_{S}$ $=D_{S},\left.D\right|_{S}: S \rightarrow S$ and $\left.D\right|_{S}$ may be extended to an element $\hat{D}$ of $\mathscr{D}_{1}$. Then $D=(D-\hat{D})+\hat{D}$ where $D-\hat{D} \in \mathfrak{D}_{2}$ and $\hat{D} \in \mathfrak{D}_{1}$. Hence, $\mathfrak{D}(A)=\mathfrak{D}_{1}$ $+\mathfrak{D}_{2}$. Since each element of $\mathfrak{D}(S)$ can be extended to an element of $\mathfrak{D}_{1}$, the complete reducibility of $\mathfrak{D}_{1}$ on $A$ follows from that of $\mathfrak{D}(S)$ on $S$. The latter result holds by [1, Theorem 5]. Again since $R \subseteq Z$, any linear transformation of $R$ is a derivation and can be extended to an element of $\mathfrak{D}_{2}$. Hence, that $\mathfrak{D}_{2}$ acts completely reducibly on $A$ follows immediately and $\mathfrak{D}(A)$ does also.

Conversely, suppose that $\mathfrak{D}(A)$ acts completely reducibly on $A$ and let $N$ be the nil-radical of $A$. We claim that $J(x, y, z)=0$ for all $x, y \in A, z \in N$ and this will hold if each term on the right-hand side of (4) is 0 . Let

$$
\begin{aligned}
& \mathfrak{D}=\langle D(z, x) ; \text { for all } z \in N, x \in A\rangle \text { and } \\
& K=\langle y D(z, x) ; \text { for all } z \in N, x, y \in A\rangle .
\end{aligned}
$$

Since $N$ is characteristic in $A$ by [2, Theorem 14], $\mathfrak{D}$ is an ideal in $\mathfrak{D}(A)$ by (3). Then

$$
(y D(z, x)) D=y[D(z, x), D]+(y D) D(z, x) \in K \quad \text { for all } y, x \in A, z \in N
$$


and, therefore, $K$ is a characteristic subspace of $A$. Let $B$ be a $\mathscr{D}(A)$-invariant complementary subspace of $K$ in $A$. If $y \in B$, then $y D(z, x) \in B \cap K=0$. Hence, $K=\langle y D(z, x)$; all $z \in N, x \in A, y \in K\rangle$. For each $z \in N, R_{z}$ is in the radical of the multiplication algebra $A^{*}$ of $A$ by [2, Theorem 2]; hence, $D(z, x)$ is in this radical and the Lie algebra $\mathfrak{L}(\mathfrak{D})$ generated by $\mathfrak{D}$ consists of nilpotent linear transformations. Suppose that $K \neq 0$ and let $J=\{y \in K$; $y D(z, x)=0$ for all $z \in N, x \in A\}$. From the condition on $\mathfrak{L}(\mathfrak{D}), J \neq 0$ by Engel's theorem. Furthermore, $J$ is $\mathfrak{D}(A)$-invariant since

$$
(y D) D(z, x)=-y D(z D, x)-y D(z, x D)+(y D(z, x)) D=0
$$

for all $D \in \mathfrak{D}(A), z \in N, y \in K$ and $x \in A$ using (3). Let $H$ be a $\mathfrak{D}(A)$ invariant complementary subspace to $J$ in $K$. For each $k \in K, k D(z, x) \in H$; hence, $K \subseteq H$ and $J=0$. Hence, $K=0$. If $K_{1}=\langle x D(y, z)$; all $x, y \in A, z$ $\in N\rangle$, then the same argument just used yields that $K_{1}=0$. Now let

$$
K_{2}=\langle z D(x, y) ; \text { all } z \in N, x, y \in A\rangle
$$

and note that $K_{2}$ is $\mathfrak{D}(A)$-invariant. Let $B_{2}$ be a $\mathfrak{D}(A)$-invariant complementary subspace of $K_{2}$ in $A$. If $z \in B_{2} \cap N$, then $z D(x, y) \in B_{2} \cap K_{2}=0$; hence, $K_{2}=\left\langle z D(x, y)\right.$; all $\left.z \in K_{2}, x, y \in A\right\rangle$. From (4),

$$
(x y) z+(y z) x+(z x) y=(z x) y-(z y) x+z(x y) \text { for all } x, y \in A, z \in N
$$

since $y D(z, x)=x D(y, z)=0$. Hence, $(x y) z=0$. Then

$$
0=x D(y, z)=(x y) z-(x z) y+x(y z)
$$

yields that

$$
R_{x} R_{y}=-R_{y} R_{x} \text { on } N .
$$

Let $S$ be the natural representation of $A$ on $N$. Then $S_{x} S_{y}=-S_{y} S_{x}$ and $\left(S_{x}\right)^{2}$ $=0$ for all $x \in A$. Then by [2, Theorem 2], $S_{x}$ lies in the radical of the multiplication algebra $S(A)^{*}$ of $S(A)$. Hence, if $\mathfrak{D}_{2}=\langle D(x, y)$ restricted to $N$; all $x, y \in A\rangle$, then $\mathfrak{L}\left(\mathfrak{D}_{2}\right)$ consists of nilpotent linear transformations. Let $J_{2}=\left\{z \in K_{2} ; z D(x, y)=0\right.$ for all $\left.x, y \in A\right\}$ and suppose that $K_{2} \neq 0$. Then $J_{2} \neq 0$ by Engel's theorem. Furthermore, $J_{2}$ is $\mathfrak{D}(A)$-invariant and we let $H_{2}$ be a $\mathfrak{D}(A)$-invariant complementary subspace of $J_{2}$ in $K_{2}$. If $k \in K_{2}$, then $k D(x, y) \in H_{2}$; hence, $K_{2} \subseteq H_{2}$ and $J_{2}=0$. Therefore, $K_{2}=0$. From (4), $J(x, y, z)=0$ for all $z \in N, x, y \in A$ and $N$ is contained in the $J$-nucleus of $A$. Hence, for all $z \in N, R_{z}$ is a derivation of $A$. Let

$$
K_{3}=\langle x z ; \text { all } x \in A, z \in N\rangle
$$

and note that $K_{3}$ is $\mathscr{D}(A)$-invariant. Let $B_{3}$ be a $\mathfrak{D}(A)$-invariant complementary subspace to $K_{3}$ in $A$ and let $x \in B_{3}$. Hence, $x z \in K_{3} \cap B_{3}=0$ and $K_{3}=$ $\left\langle x z\right.$; all $\left.x \in K_{3}, z \in N\right\rangle$. Let $z \in B_{3} \cap N$. Then $x z \in K_{3} \cap B_{3}=0$ and $K_{3}$ $=\left\langle x z ;\right.$ all $\left.z, x \in K_{3}\right\rangle$. Now $K_{3}=K_{3}^{2}$ and since $K_{3} \subseteq N, K_{3}=0$. Hence, $N \subseteq Z$. If $N \neq R$, then $A / N$ contains a minimal nonzero ideal $E / N$ such that $E / N$ is solvable. By the remarks preceding this proof, $E^{2} \subseteq N$, and since $N \subseteq Z, E$ is nilpotent, a contradiction. Hence $R=N \subseteq Z$. 
2. The Lie triple system case. A vector space $A$ with trilinear composition $(a, b, c) \rightarrow[a, b, c]$ satisfying

$$
\begin{aligned}
{[a, a, b] } & =0, \\
{[a, b, c]+[b, c, a]+[c, a, b] } & =0,
\end{aligned}
$$

and

$$
[a, b,[x, y, z]]=[[a, b, x], y, z]+[x,[a, b, y], z]+[x, y,[a, b, z]]
$$

is called a Lie triple system. For $x, y \in A$, the mapping $D(x, y): z \rightarrow[x, y, z]$ is a derivation of $A$ and these are called inner derivations.

Proof of the Lie triple system CaSe. Suppose that $R \subseteq Z$. Let $S$ be a semisimple subsystem complementary to $R$ [3, Theorem 2.21]. Let

$$
\begin{aligned}
& \mathfrak{D}_{1}=\{D \in \mathfrak{D}(A) ; D: S \rightarrow S, D: R \rightarrow 0\} \text { and } \\
& \mathscr{D}_{2}=\{D \in \mathfrak{D}(A) ; D: S \rightarrow 0\} .
\end{aligned}
$$

We claim that $\mathscr{D}(A)$ is the direct sum of ideals $\mathscr{D}_{1}$ and $\mathfrak{D}_{2}$, each of which acts completely reducibly on $A$. Then $\mathfrak{D}(A)$ will act this way on $A$. Let $D \in \mathfrak{D}(A)$. Then $D$ restricted to $S$ can be extended to a derivation $\hat{D}$ of $A$, where

$$
\hat{D}=\sum D\left(x_{i}, y_{i}\right),
$$

where $x_{i}, y_{i} \in A$ by [3, Theorem 2.18]. Since $R \subseteq Z, x_{i}, y_{i}$ may be assumed to be in $S$. Then $D: S \rightarrow S$ and, using (5) and (6), $\hat{D}: R \rightarrow 0$; hence, $D=(D-\hat{D})+\hat{D}$ where $D-\hat{D} \in \mathscr{D}_{2}$ and $\hat{D} \in \mathscr{D}_{1}$. Therefore, $\mathscr{D}(A)=\mathscr{D}_{1}$ $\oplus \mathfrak{D}_{2}$. In the same manner, each element in $\mathscr{D}(S)$ may be extended to an inner derivation of $A$ and, since $R \subseteq Z$, this extension is in $\mathscr{D}_{1}$. Hence, to show that $\mathfrak{D}_{1}$ acts completely reducibly on $A$, it is enough to show that $\mathscr{D}(S)$ acts this way on $S$. Since every derivation of $S$ is inner [3, Theorem 2.11] and since $S$ is the direct sum of simple ideals [3, Theorem 2.9], we may assume that $S$ is simple. This result holds by [3, Theorem 4.1]. To show that $\mathscr{D}_{2}$ acts completely reducibly on $A$, note that each linear transformation of $R$ is a derivation which, since $R \subseteq Z$, can be extended to an element of $\mathfrak{D}_{2}$. Hence, $\mathfrak{D}_{2}$ acts in the desired manner on $A$ and, therefore, $\mathscr{D}(A)$ does as well.

Conversely, suppose that $\mathscr{D}(A)$ acts completely reducibly on $A$. Let

$$
\begin{aligned}
& \mathscr{D}=\langle D(x, y) ; \text { all } x \in R, y \in A\rangle \text { and } \\
& K=\langle z D(x, y)=[x, y, z] ; \text { all } x \in R, y, z \in A\rangle .
\end{aligned}
$$

Since $R$ is $\mathfrak{D}(A)$-invariant [4, Lemma 5], $K$ is also. Let $J$ be a $\mathfrak{D}(A)$-invariant complementary subspace to $K$ in $A$. If $z \in J$, then $z D(x, y) \in K \cap J=0$. Hence, $K=\langle z D(x, y)$; all $x \in R, y \in A, z \in K\rangle$. If $z \in K, y \in J$ and $x$ $\in R \cap J$, then

$$
z D(x, y)=[x, y, z]=-[y, z, x]-[z, x, y]=[z, y, x]=0 .
$$

Hence, $K=\langle z D(x, y)$; all $z, x \in K, y \in A$. $\rangle$. Therefore, $[A, K, K]=[K, A, K]$ $=K$, and since $K \subseteq R$ and $R$ is solvable, $K=0$. Hence, $z D(x, y)=[x, y, z]$ 
$=0$ and $x \in Z$. Hence, $R \subseteq Z$ and the proof is complete.

Corollary. Let $A$ be a Malcev algebra over a field of characteristic 0 and let $T_{A}$ be the Lie triple system associated with $A$. If $\mathfrak{D}(A)$ acts completely reducibly on $A$, then $\mathfrak{D}\left(T_{A}\right)$ does also.

Note that the converse of this corollary fails as is seen by the three dimensional, nilpotent, nonabelian Lie algebra.

\section{REFERENCES}

1. W. H. Davenport, On inner derivations of Malcev algebras, Rocky Mountain J. Math. 2 (1972), no. 4, 565-568. MR 46 \#9129.

2. E. N. Kuz' min, Mal' cev algebras and their representations, Algebra i Logika 7 (1968), no. 4, 48-69 = Algebra and Logic 7 (1968), 233-244. MR 40 \#568.

3. W. G. Lister, A structure theory of Lie triple systems, Trans. Amer. Math. Soc. 72 (1952), 217-242. MR 13, 619.

4. O. Loos, Uber eine Beziehung zwischen Malcev-Algebren und Lie-Triplesystemen, Pacific J. Math. 18 (1966), 553-562. MR 33 \# 7385.

5. T. S. Ravisankar, On certain classes of Malcev algebras, J. Sci. Hiroshima Univ. Ser. A-I Math. 32 (1968), 233-236. MR 39 \#1510.

6. - On Malcev algebras, Pacific J. Math. 42 (1972), 227-234. MR 47 \# 1905.

7. A. A. Sagle, Malcev algebras, Trans. Amer. Math. Soc. 101 (1961), 426-458. MR 26 \#1343.

8. R. D. Schafer, An introduction to non-associative algebras, Pure and Appl. Math., vol. 22, Academic Press, New York, and London, 1966. MR 35 \# 1643.

9. E. L. Stitzinger, Standard and alternative algebras with completely reducible derivation algebras, Proc. Amer. Math. Soc. 43 (1974), 57-62.

10. —-, Malcev algebras with $J_{2}$-potent radical, Proc. Amer. Math. Soc. 50 (1975), 1-9.

Department of Mathematics, North Carolina State University, Raleigh, North CaroLINA 27607 